\title{
Predictors of Effectiveness of Anakinra in Systemic Juvenile Idiopathic Arthritis
}

\author{
Benedetta Saccomanno, Jessica Tibaldi ${ }^{(D)}$, Francesca Minoia (D), Francesca Bagnasco (D), \\ Angela Pistorio (1), Andressa Guariento (1), Roberta Caorsi (1), Alessandro Consolaro (1), \\ Marco Gattorno (i), and Angelo Ravelli (i)
}

ABSTRACT. Objective. To seek predictors of therapeutic response to the interleukin (IL)-1 inhibitor anakinra in children with systemic-onset juvenile idiopathic arthritis (sJIA).

Methods. The clinical charts of all patients with sJIA who were newly treated with anakinra at our center between 2004 and 2017 were reviewed retrospectively. Predictors included baseline demographic, clinical, and laboratory variables as well as previous or concomitant therapies. The effectiveness of anakinra was assessed at 1 year after treatment start. Complete clinical response (CCR) was defined as absence of fever, physician's global assessment $\leq 1$, count of active joints $\leq 1$, negative C-reactive protein, and $\geq 75 \%$ reduction of corticosteroid dose. According to the intention-to-treat principle, patients who had anakinra discontinued before 1 year for any reasons other than disease remission were classified as nonresponders. Statistics included univariate and multivariable analyses. Results. Of the 62 patients included in the study, 24 (39\%) met the criteria for CCR at 1 year, whereas $38(61 \%)$ did not. On multivariable analysis, independent correlations with achievement of CCR were identified for shorter disease duration, lower active joint count, higher ferritin level, and greater activity of systemic manifestations. The area under the curve of the model was 0.83 .

Conclusion. Our findings help to delineate the clinical profile of patients with sJIA who are more likely to benefit from IL-1 blockade. They also underscore the need for studies aimed at examining the therapeutic role of early IL-1 inhibition and to identify biomarkers predicting response to either IL-1 or IL-6 antagonists. (First Release January 15 2019; J Rheumatol 2019;46:416-21; doi:10.3899/ jrheum.180331)

\section{Key Indexing Terms: SYSTEMIC JUVENILE IDIOPATHIC ARTHRITIS INTERLEUKIN 1 INHIBITORS}

Systemic-onset juvenile idiopathic arthritis (sJIA) accounts for $5-15 \%$ of all chronic arthritis seen in children in Europe and North America ${ }^{1}$, but is much more common in some

From the Università degli Studi di Genova, and the Istituto Giannina Gaslini, Genoa; Fondazione Institute for Research and Health Care (IRCCS) Ca' Granda, Ospedale Maggiore Policlinico, Milan, Italy; Instituto de Criança - Faculty of Medicine of the University of São Paulo (FMUSP), São Paulo, Brazil.

$M G$ has received speaking or consultant fees from Sobi.

B. Saccomanno, MD, Research Fellow, Università degli Studi di Genova; J. Tibaldi, MD, PhD Student, Università degli Studi di Genova; F. Minoia, MD, Dirigente Medico, Fondazione IRCCS Ca' Granda, Ospedale Maggiore Policlinico; F. Bagnasco, PhD, Biostatistician, Istituto Giannina Gaslini; A. Pistorio, MD, PhD, Dirigente Medico, Istituto Giannina Gaslini; A. Guariento, MD, Research Fellow, Istituto Giannina Gaslini, and Instituto de Criança-FMUSP; R. Caorsi, MD, Dirigente Medico, Istituto Giannina Gaslini; A. Consolaro, MD, PhD, Assistant Professor, Università degli Studi di Genova and Istituto Giannina Gaslini;

M. Gattorno, MD, Dirigente Medico, Istituto Giannina Gaslini; A. Ravelli, MD, Professor of Pediatrics, Università degli Studi di Genova and Istituto Giannina Gaslini.

Dr. Saccomanno and Dr. Tibaldi contributed equally to the study.

Address correspondence to Dr. A. Ravelli, Clinica Pediatrica e

Reumatologia, Istituto G. Gaslini, Via G. Gaslini 5, 16147 Genoa, Italy.

E-mail: angeloravelli@gaslini.org

Accepted for publication September 20, 2018.

\section{INTERLEUKIN 1 ANAKINRA}

Asian countries, particularly India, Thailand, and Japan, where frequencies as high as $50 \%$ have been reported ${ }^{2}$. It is quite distinct from the other categories of JIA because of the association of arthritis with a severe systemic illness ${ }^{3,4}$. By the current International League of Associations for Rheumatology (ILAR) criteria, the diagnosis of sJIA requires the presence of arthritis accompanied or preceded by quotidian fever of at least 2 weeks' duration, plus 1 or more of the following: typical evanescent, non-fixed erythematous rash; hepatomegaly or splenomegaly; generalized lymphadenopathy; or serositis 5 .

It is a common view that sJIA is the most severe form of childhood arthritis and the most difficult to treat ${ }^{6}$. Until recently, sJIA was considered a therapeutic orphan, because the most effective treatment was glucocorticoids (GC), whose prolonged administration exposes children to their serious side effects, which include growth failure and osteoporosis. Synthetic disease-modifying antirheumatic drugs (DMARD) such as methotrexate (MTX) have limited efficacy for arthritis and little or no effect on systemic symptoms. Inadequate responses have also been observed with the anti-tumor necrosis factor (TNF) medications $7,8,9,10$, although these agents may be effective in the later afebrile

Personal non-commercial use only. The Journal of Rheumatology Copyright (c) 2019. All rights reserved 
disease stage, in which chronic arthritis predominates ${ }^{11,12}$.

The demonstration of the key pathogenetic role of interleukin 1 (IL-1) in a seminal experimental study ${ }^{13}$ has opened the way to the successful treatment of sJIA with biologic DMARD that selectively antagonize this cytokine ${ }^{6}$. However, not all patients were responsive to IL-1 blockade $14,15,16,17$. Identification of patients who have a greater likelihood of benefiting from IL-1 inhibition could help select candidates for the early introduction of this therapy. Further, it might aid in establishing the respective indications of the biologic medications that target IL-1 or IL-6, both of which were found to be efficacious in SJIA $^{6,18}$.

A number of potential predictors of the therapeutic effectiveness of IL-1 inhibitors have been reported, which include less severe joint disease and increased white blood cell or neutrophil count ${ }^{16,19}$, shorter disease duration ${ }^{20}$, older age at disease onset $^{21}$, and use of IL-1 blockade as first-line therapy, particularly in patients with new-onset disease and not yet exposed to GC or DMARD ${ }^{21,22}$. However, because the experience gained so far is still limited, there is a need for further data to better characterize the profile of patients with sJIA who are more likely to respond to IL-1 blockade.

The aim of our present study was to seek predictors of therapeutic response in children with sJIA treated with the IL-1 inhibitor anakinra at our center.

\section{MATERIALS AND METHODS}

Patient selection. We conducted a retrospective review of the clinical and laboratory features of all patients with sJIA who were newly treated with anakinra at our center between 2004 and 2017. Patients were diagnosed as having SJIA if they met the ILAR classification criteria for this JIA category ${ }^{5}$. Patients with monogenic periodic fevers or other autoinflammatory diseases were excluded. A previous or concomitant treatment was allowed with nonsteroidal antiinflammatory drugs, systemic or intraarticular corticosteroids, synthetic DMARD (e.g., MTX), or biologic DMARD other than anakinra. Patients who had treatment with IL-1 antagonists started elsewhere before our first observation were excluded. Patients given IL-1 inhibitors for the treatment of macrophage activation syndrome (MAS) were also excluded from the study.

The study protocol was approved by the Regional Ethics committee of Liguria, Genoa, Italy on the June 18, 2018 (meeting minutes no. 10/2018).

Assessment of baseline predictors. These demographic or clinical variables were recorded for each patient at treatment start: age, sex, disease duration, previous and concomitant therapies, dose of anakinra, physician's global assessment (PGA) of overall disease activity on a $0-10$ visual analog scale $(0=$ no activity; $10=$ maximum activity), count of active joints (defined as reported $^{23}$ ), fever, rash, hepatomegaly, splenomegaly, generalized lymphadenopathy, and serositis. Laboratory variables included white blood cell count, neutrophil count, hemoglobin, platelet count, erythrocyte sedimentation rate, $\mathrm{C}$-reactive protein (CRP), fibrinogen, and ferritin. To obtain a quantitative measurement of the activity of systemic disease, a systemic manifestation score (SMS) was devised by weighting extraarticular features: fever $=1$ point if $37-38^{\circ} \mathrm{C}, 2$ points if $38-39^{\circ} \mathrm{C}, 3$ points if $39-40^{\circ} \mathrm{C}, 4$ points if $>40^{\circ} \mathrm{C}$; rash $=1$ point; generalized lymphadenopathy $=1$ point; hepatomegaly and $/$ or splenomegaly $=1$ point; serositis $=1$ point; anemia (hemoglobin $<9 \mathrm{~g} / \mathrm{dl})=1$ point; platelet count $>\times 10^{9} / 1$ or ferritin $>500 \mathrm{ng} / \mathrm{ml}=1$ point (Table 1 ). It was decided to assign a greater weight to fever owing to its greater effect on a child's well-being and major importance in driving treatment decisions. The SMS ranges from 0 to 10 , where $0=$ absence of systemic manifestations and $10=$ maximum activity of systemic manifestations.
Assessment of treatment response. The effectiveness of anakinra was assessed in all patients at 1 year after treatment start. A complete clinical response (CCR) was defined according to the following 5 criteria, which should all be present: (1) absence of fever; (2) PGA of the overall disease activity $\leq 1$; (3) count of active joints $\leq 1$; (4) negative CRP; and (5) $\geq 75 \%$ reduction of dosage of corticosteroid therapy from baseline. In line with the intention-to-treat principle, patients who discontinued anakinra before reaching 1 year or had treatment for any reasons other than disease remission were classified as nonresponders. Patients who discontinued anakinra before 1 year because of disease remission and had remained in such a state until 1 year were considered responders.

Assessment of adverse events. All clinically significant anakinra-related adverse events observed during the first year of treatment were recorded for each patient. Adverse events that led to treatment discontinuation were registered.

Statistics. Descriptive statistics were reported as medians and interquartile ranges (IQR) for continuous variables and as absolute frequencies and percentages for categorical variables. Comparisons of quantitative variables between 2 groups were made by Mann-Whitney U test, whereas categorical data were compared by chi-square test or by Fisher's exact test, as appropriate.

Multivariable logistic regression analysis was performed, entering explanatory variables that showed significant results in univariate tests $(\mathrm{p}<0.05)$ or were considered a priori to be of foremost importance for the study outcome, with CCR as the outcome variable. Cases with missing variables were excluded from the analysis. Before the application of logistic regression procedures, some quantitative variables were dichotomized to binary variables, using the cutpoints obtained through the receiver-operating characteristic (ROC) curve analysis. Variables that were significantly associated with the study outcomes were identified using a backward selection procedure. Possible explanatory variables assessed were all those listed in Table 2. The effect was expressed in OR, and 95\% CI were calculated; statistical significance was tested by likelihood ratio test. The area under the ROC curve of the best-fitting model was used as an indicator of the predictive ability of the model.

All statistical tests were 2-sided; p values $<0.05$ were considered significant. The statistical package used was Stata (Stata Corp., Release 11).

\section{RESULTS}

In the study time frame, a total of 116 patients were treated with IL-1 antagonists at our center. Fifty-four (46.5\%) of them were excluded from the study because they had an autoinflammatory syndrome $(\mathrm{n}=31)$, had started anakinra elsewhere before our first observation $(n=18)$, had received a different IL-1 inhibitor (i.e., canakinumab, $n=3$ ), or were given anakinra specifically for the management of MAS

Table 1. Systemic manifestation score.

\begin{tabular}{lc}
\hline Clinical manifestation & Points \\
\hline Fever & \\
$\quad 37-38^{\circ} \mathrm{C}$ & 1 \\
$38-39^{\circ} \mathrm{C}$ & 2 \\
$39-40^{\circ} \mathrm{C}$ & 3 \\
$>40^{\circ} \mathrm{C}$ & 4 \\
Evanescent erythematous rash & 1 \\
Generalized lymphadenopathy & 1 \\
Hepatomegaly and/or splenomegaly & 1 \\
Serositis & 1 \\
Anemia (hemoglobin $<9 \mathrm{~g} / \mathrm{dl}$ ) & 1 \\
Platelet count $>600 \times 10^{9} / 1$ or ferritin $>500 \mathrm{ng} / \mathrm{ml}$ & 1 \\
\hline
\end{tabular}


Table 2. Baseline demographic and clinical features of the study patients considered as a whole and divided by response to IL-1 inhibition.

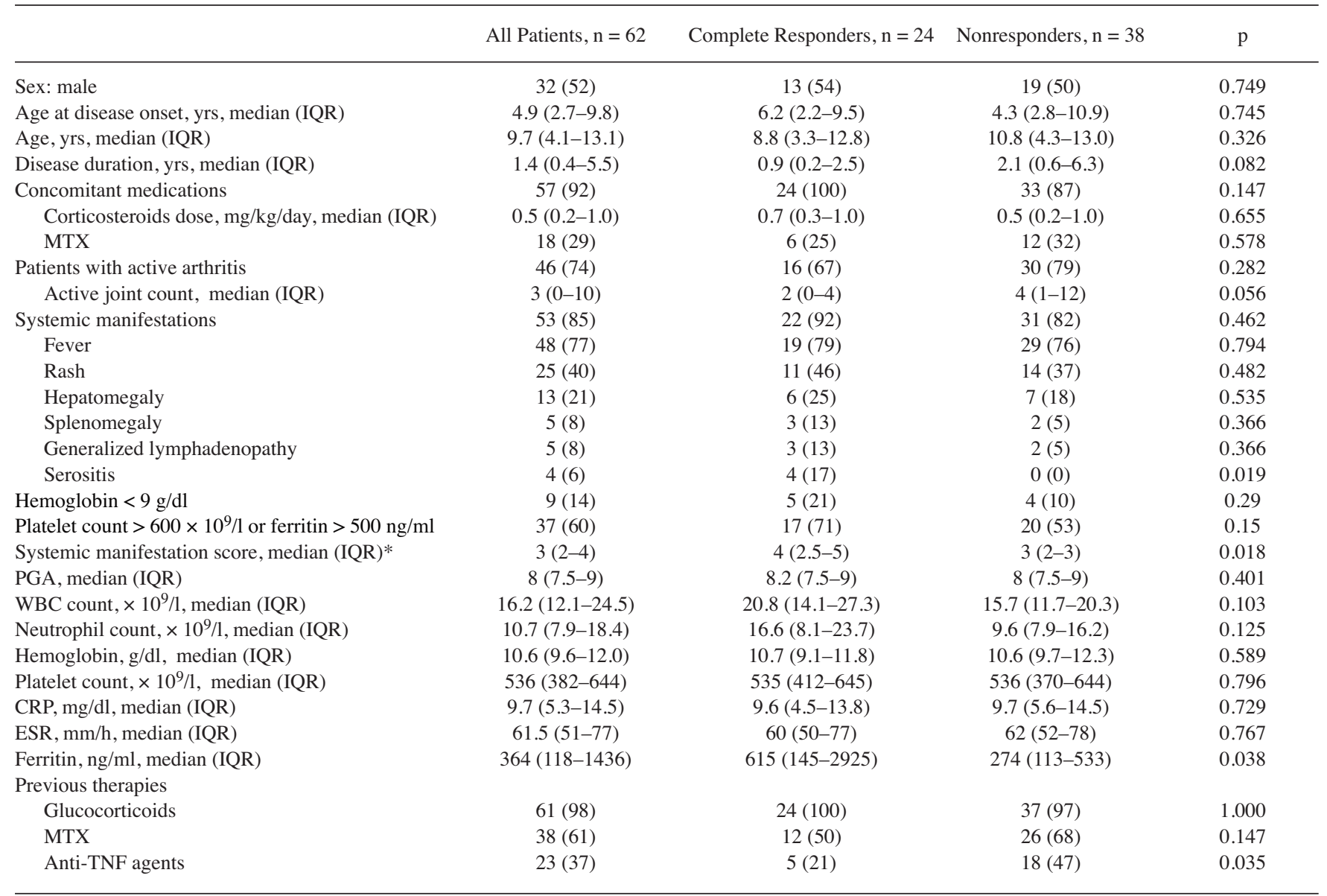

Data are represented as n $(\%)$, unless otherwise indicated. *Systemic manifestation score (0-10 points). IQR: interquartile range; PGA: physician's global assessment of disease activity; MTX: methotrexate; CRP: C-reactive protein; ESR: erythrocyte sedimentation rate; WBC: white blood cell; anti-TNF: tumor necrosis factor antagonists; IL: interleukin.

$(\mathrm{n}=2)$. The main demographic, clinical, and laboratory features of the 62 patients included in the study are presented in Table 2. The proportion of males and females was comparable and the median disease duration at treatment initiation was 1.4 years (IQR 0.4-5.5). The median initial dosage of anakinra was $1.5 \mathrm{mg} / \mathrm{kg}$ daily (IQR $1.20-1.87$ ). Twenty-two of the study patients had been included in a previous analysis published by our group ${ }^{16}$.

At treatment start, patients had on average a high level of disease activity, as demonstrated by the presence of fever in $77 \%$ of them, concomitant or previous corticosteroid therapy in $92 \%$ and $98 \%$, respectively, and the high median values of PGA, active joint count, and acute-phase reactants. Twenty-three patients were receiving concomitant synthetic DMARD, including MTX, oral cyclosporine, or azathioprine. Most patients also had refractory disease; 42 had previously received treatment with synthetic DMARD and 23 with biologic DMARD other than anakinra (1 with tocilizumab) with inadequate response. The patients who were receiving azathioprine had this medication prescribed elsewhere. Azathioprine was discontinued at the start of anakinra.
At 1 year after the start of anakinra, 24 patients (39\%) met the criteria for CCR, whereas 38 patients $(61 \%)$ did not. Of the 38 patients who were nonresponders, 17 had the medication withdrawn before the study endpoint for inefficacy (8 patients), adverse events or intolerance (7 patients), and difficulty in the delivery of the drug by the local pharmacy ( 2 patients). The 2 latter patients were kept in the analysis as nonresponders in line with the intentionto-treat principle. The median time to withdrawal was 0.76 months (IQR 0.39-1.25). Of the 24 patients who had CCR at 1 year, 18 were still receiving anakinra but had discontinued all other medications, and 6 were still taking low-dose corticosteroids and/or synthetic DMARD in combination with anakinra.

The comparison of the baseline features between patients with and without CCR at 1 year showed that the group with more favorable treatment outcome had shorter disease duration, lower number of active joints, greater frequency of serositis, higher SMS and ferritin level, and had received anti-TNF medications less frequently (Table 2). The median (IQR) dose of anakinra at 1 year was $1.54(1-1.99) \mathrm{mg} / \mathrm{kg} / \mathrm{day}$.

Personal non-commercial use only. The Journal of Rheumatology Copyright @ 2019. All rights reserved. 
The median (IQR) dose of prednisone at the beginning of treatment was $0.7(0.3-1.0) \mathrm{mg} / \mathrm{kg} /$ day and $0.5(0.2-1.0)$ $\mathrm{mg} / \mathrm{kg} /$ day in responders and nonresponders, respectively, and at 1 year was $0(0-0.14) \mathrm{mg} / \mathrm{kg} / \mathrm{day}$ and $0(0-0.16) \mathrm{mg} / \mathrm{kg} / \mathrm{day}$ in responders and nonresponders, respectively.

For the multivariable analysis, complete data were available for 61 patients. The best-fitting model obtained through logistic regression procedures, in which the achievement of CCR at 1 year was the dependent variable, is presented in Table 3. Independent correlations of baseline variables with the achievement of CCR were identified for shorter disease duration, lower active joint count, higher ferritin level, and greater SMS. The area under the curve of the model was 0.83 . To evaluate the utility of weighting the item "fever" in the SMS, we recalculated the score by giving all fevers a value of 1 and repeated the statistical analyses using this simplified version of the score. After this change, the SMS was no longer associated with the outcome in multivariable analysis (results not shown).

The evaluation of therapeutic effectiveness at 3 months after treatment start showed that 18 of 57 patients (31.6\%) had achieved CCR. In multivariable analysis, shorter disease duration and greater SMS remained independently associated with the study outcome, whereas count of active joints and ferritin level were replaced by neutrophil count and CRP (results not shown). Of the 62 study patients, 10 had anakinra started within 3 months after disease onset. Of them, 6 had CCR at 3 months and 7 had CCR at 1 year. During the period of observation, 8 patients $(13 \%)$ had 1 or more disease flares requiring treatment adjustment or intensification.

Adverse events were reported for 10 patients: 5 developed MAS, which led to treatment discontinuation in 3 cases. However, 1 more recent case of MAS recovered after doubling the dosage of anakinra from $100 \mathrm{mg}$ in a single daily administration to $100 \mathrm{mg}$ twice a day. Two patients were discontinued from treatment for elevation of liver enzymes (one of them was also taking MTX) and 3 patients could not continue therapy because of severe injection site reactions to anakinra. Of the 2 patients who had hypertransaminasemia, 1 had acute liver failure and 1 only a moderate elevation of liver enzymes. Hepatotoxicity resolved in both patients after withdrawal of anakinra.

Table 3. Best-fitting model obtained through logistic regression procedures.*

\begin{tabular}{lcc}
\hline Baseline Explanatory Variable & OR $(95 \% \mathrm{CI})$ & $\mathrm{p}^{\mathfrak{1}}$ \\
\hline Disease duration $\leq 3.9$ yrs & $6.78(1.30-35.27)$ & 0.012 \\
Active joint count $\leq 10$ & $8.25(1.26-53.91)$ & 0.012 \\
Ferritin $>444 \mathrm{ng} / \mathrm{ml}$ & $4.75(1.16-19.50)$ & 0.020 \\
Systemic manifestation score $>3$ & $6.44(1.38-24.62)$ & 0.007 \\
\hline
\end{tabular}

* Complete clinical response was the dependent variable. Complete data were available for 61 patients. The area under the receiver-operating characteristic curve of the model is $0.83 .{ }^{£}$ By likelihood ratio test.

\section{DISCUSSION}

We found that $39 \%$ of our patients with sJIA achieved a CCR at 1 year after start of treatment with anakinra. Although the criteria used in our study to define therapeutic response are a bit looser than those of the Wallace, et $a^{24,25}$ and Juvenile Arthritis Disease Activity Score (JADAS) ${ }^{26}$ definitions of inactive disease, they are nonetheless quite stringent. They required the absence of fever, a maximum value of both PGA and active joint count of 1 , and a negative CRP. In addition, the dosage of corticosteroid medications had to be reduced by at least $75 \%$ from baseline. These criteria would correspond to no less than the state of low (or minimal) disease activity $26,27,28$, which is regarded as a qualified therapeutic target in patients with JIA ${ }^{29}$. Note that a disease activity tool specific to sJIA is not yet available ${ }^{30}$. We did not assess therapeutic effectiveness in percentage improvement in clinical signs and symptoms because current clinical practice mandates good overall disease control ${ }^{31}$. Further, we evaluated treatment outcome at 1 year to minimize the influence of initial high-dose corticosteroid administration.

The rate of CCR observed in our study is in the low range of the percentage of excellent response (either inactive disease or complete response) reported in previous analyses of the effectiveness of IL-1 antagonists, which varies from $31 \%$ to $85 \%^{14,19,20,21,22}$. The wide disparity in response figures across studies may depend on differences in disease activity and severity, therapeutic protocols, timeline of response evaluation, concomitant therapies, and criteria used to assess response.

However, the varying susceptibility to IL-1 inhibition may also be explained by the pathophysiologic heterogeneity of sJIA. The observation that only half of patients responded well led to speculation that sJIA might contain 2 biologically distinct subgroups, only 1 of which is dependent on IL- 1 . This subgroup had fewer affected joints and increased neutrophil count ${ }^{16}$. Other investigators have found evidence that IL-1 blockade may be more effective for systemic features than for articular manifestations of the disease $\mathrm{e}^{32}$.

Shimizu and colleagues ${ }^{33}$ delineated 2 distinct sJIA patient subsets based on their serum IL-6 and IL-18 levels: an IL-6 dominant and an IL-18 dominant. The IL-6-dominant subset had a more severe polyarthritis and higher serum levels of matrix metalloproteinase, whereas the IL-18-dominant subset was more prone to develop MAS. Owing to the recent reports of the effectiveness of anakinra in cases of MAS refractory to conventional therapies ${ }^{34}$, it might be inferred that the second subset may be more susceptible to IL-1 blockade.

In this respect, it should be noted that in the studies by Nigrovic, et $a l^{21}$ and Pardeo, et $a l^{20}$, the median level of ferritin, which is the hallmark laboratory abnormality of MAS $^{35}$, tended to be higher in patients who had a complete response or reached inactive disease than in those who did not (3008 vs $1329 \mathrm{ng} / \mathrm{ml}$ and 1506 vs $306 \mathrm{ng} / \mathrm{ml}$, respectively). Although the difference was not significant, perhaps

Personal non-commercial use only. The Journal of Rheumatology Copyright @ 2019 . All rights reserved. 
because of the small size of the study samples, this observation suggests that patients with more prominent activation of the monocyte/macrophage activation syndrome are more prone to respond to IL-1 inhibition.

Another explanation for the inconsistent effectiveness of IL-1 inhibition could be the timing of therapy. Nearly all patients included in earlier open studies and in randomized clinical trials had longstanding disease when treatment with IL-1 blocking agents was initiated. These characteristics may account for the partial or absent responses seen in a significant minority of patients. More favorable outcomes were obtained with the use of IL-blockade as first-line therapy, particularly in patients with new-onset disease and not yet exposed to corticosteroids or other DMARD ${ }^{21,22}$. Many patients achieved inactive disease rapidly and were able to stop IL-1 inhibitors within 1 year, with sustained remission during followup 22 . Importantly, a significant reduction was observed in the proportion of children whose disease progressed to the chronic polyarthritis stage ${ }^{21}$. The differential clinical responses in early versus late disease have led to the theory of a "window of opportunity" in which sJIA pathophysiology can be altered by early treatment with IL-1 inhibitors to secure rapid remission of systemic disease and to avoid the occurrence of chronic arthritis ${ }^{36}$.

The results of our predictor analysis showed that the achievement of CCR with anakinra was associated with shorter disease duration, lower active joint count, higher ferritin level, and greater activity of systemic manifestations. Notably, the frequency of CCR in the 10 patients who had anakinra started within 3 months after disease onset was $60 \%$ at 3 months and $70 \%$ at 1 year. These findings are in keeping with the window of opportunity hypothesis because they suggest that IL-1 blockade may be more effective in the earlier stages of sJIA, when the disease is characterized by more prominent systemic manifestations, greater activation of the monocyte/macrophage system, and less extended joint disease. Indirect support for this hypothesis is also provided by our observation of a weaker response to IL- 1 inhibition among patients previously treated with anti-TNF medications, who had, on average, a more spread joint disease and a longer disease history.

Our study should be interpreted in the light of some caveats, the chief of which is its retrospective nature. A retrospective data collection is subject to missing and possibly erroneous data. Because patients were not randomized, we cannot exclude that the responder group included a greater proportion of cases with more benign disease. It is well known that around $40 \%$ of patients with sJIA have a monocyclic course with spontaneous remission ${ }^{1,3}$. Thus, the results of open studies on patients with early disease may be biased toward patients destined to a milder course. However, our sample was composed of consecutive patients seen in a tertiary care referral center, most of whom were resistant to or dependent on corticosteroid therapy or were refractory to synthetic DMARD. We acknowledge that the standard dose of anakinra of 1-2 $\mathrm{mg} / \mathrm{kg}$ day used in our study might have been insufficient in some patients. Some clinical experiences have shown that increasing IL-1 blockade dosage will often treat sJIA $^{21}$. We recognize that the conclusions from our study may not be universal for patients with sJIA because almost one-third of the patients were excluded from analysis. It might be surprising that only 2 patients received IL-1 blockade for MAS. This low number was largely due to most patients being followed many years ago, when the therapeutic role of anakinra in MAS had not yet been demonstrated. The same reason explains why anakinra was discontinued in some patients who developed MAS. In the past, concern was raised that anakinra could trigger MAS in children with sJIA ${ }^{37}$. All our 3 patients who had anakinra stopped for MAS had developed this complication within 2 weeks after the start of this medication. One of these patients did not have a recurrence of MAS after reintroduction of anakinra 7 months later. Two other patients had MAS at 1 and 3 months after the beginning of treatment and were not discontinued. It is increasingly realized that increasing the dose of biologic medications will often abate $\mathrm{MAS}^{34}$. Currently, the administration of anakinra has become a standard of care at our center for patients with sJIA-associated MAS that is severe or refractory to conventional therapies. Owing to the retrospective design of our study and the lack of standardized clinical assessments in many patients, we could not apply the current accepted outcome measures to define response, such as the American College of Rheumatology pediatric response criteria ${ }^{38}$ or the Wallace or JADAS definitions of inactive disease ${ }^{24,25,26,27,28}$, but used a composite score not yet validated.

We found that in our patients, sJIA response to anakinra was associated with shorter disease duration, less severe polyarthritis, higher ferritin levels, and more pronounced systemic manifestations. These findings may help to define the profile of patients with sJIA who are more likely to benefit from IL-1 blockade. Additional investigations are needed to further elucidate the pathophysiology of sJIA and to explore the use of IL-1 antagonists early in the disease course. Future studies should also seek to identify biomarkers predicting response to either IL-1 or IL-6 inhibitors.

\section{REFERENCES}

1. Ravelli A, Martini A. Juvenile idiopathic arthritis. Lancet 2007;369:767-78.

2. Consolaro A, Ravelli A. Unraveling the phenotypic variability of juvenile idiopathic arthritis across races or geographic areas - key to understanding etiology and genetic factors? J Rheumatol 2016;43:683-5

3. De Benedetti F, Schneider R. Systemic juvenile idiopathic arthritis. In: Cassidy JT, Petty RE, Laxer RM, Lindsley CB, editors. Textbook of pediatric rheumatology, 6th ed. Philadelphia: Elsevier Saunders; 2011:236-48.

4. Ravelli A. Handbook of juvenile idiopathic arthritis. Urdorf, Switzerland: Adis; 2015:1-124.

5. Petty RE, Southwood TR, Manners P, Baum J, Glass DN, Goldenberg $\mathrm{J}$, et al. International League of Associations for

Personal non-commercial use only. The Journal of Rheumatology Copyright $\subset$ 2019. All rights reserved. 
Rheumatology classification of juvenile idiopathic arthritis: second revision, Edmonton, 2001. J Rheumatol 2004;31:390-2.

6. Giancane G, Minoia F, Davi S, Bracciolini G, Consolaro A, Ravelli A. IL-1 inhibition in systemic juvenile idiopathic arthritis. Front Pharmacol 2016;7:467.

7. Quartier P, Taupin P, Bourdeaut F, Lemelle I, Pillet P, Bost M, et al. Efficacy of etanercept for the treatment of juvenile idiopathic arthritis according to the onset type. Arthritis Rheum 2003; 48:1093-101.

8. Horneff G, Schmeling H, Biedermann T, Foeldvari I, Ganser G, Girschick HJ, et al. The German etanercept registry for treatment of juvenile idiopathic arthritis. Ann Rheum Dis 2004;63:1638-44.

9. Kimura Y, Pinho P, Walco G, Higgins G, Hummell D, Szer I, et al. Etanercept treatment in patients with refractory systemic onset juvenile rheumatoid arthritis. J Rheumatol 2005;32:935-42.

10. Solari N, Palmisani E, Consolaro A, Pistorio A, Viola S, Buoncompagni A, et al. Factors associated with achievement of inactive disease in children with juvenile idiopathic arthritis treated with etanercept. J Rheumatol 2013;40:192-200.

11. Lovell DJ, Ruperto N, Goodman S, Reiff A, Jung L, Jarosova K, et al. Adalimumab with or without methotrexate in juvenile rheumatoid arthritis. N Engl J Med 2008;359:810-20.

12. Giannini EH, Ilowite NT, Lovell DJ, Wallace CA, Rabinovich CE, Reiff A, et al. Long-term safety and effectiveness of etanercept in children with selected categories of juvenile idiopathic arthritis. Arthritis Rheum 2009;60:2794-804.

13. Pascual V, Allantaz F, Arce E, Punaro M, Banchereau J. Role of interleukin-1 (IL-1) in the pathogenesis of systemic onset juvenile idiopathic arthritis and clinical response to IL-1 blockade. J Exp Med 2005;201:1479-86.

14. Quartier P, Allantaz F, Cimaz R, Pillet P, Messiaen C, Bardin C, et al. A multicentre, randomised, double-blind, placebo-controlled trial with the interleukin-1 receptor antagonist anakinra in patients with systemic-onset juvenile idiopathic arthritis (ANAJIS trial). Ann Rheum Dis 2011;70:747-54.

15. Swart JF, Barug D, Mohlmann M, Wulffraat NM. The efficacy and safety of interleukin-1-receptor antagonist anakinra in the treatment of systemic juvenile idiopathic arthritis. Expert Opin Biol Ther 2010;10:1743-52.

16. Gattorno M, Piccini A, Lasiglie D, Tassi S, Brisca G, Carta S, et al. The pattern of response to anti-interleukin- 1 treatment distinguishes two subsets of patients with systemic-onset juvenile idiopathic arthritis. Arthritis Rheum 2008;58:1505-15.

17. Lequerre T, Quartier P, Rosellini D, Alaoui F, De Bandt M, Mejjad $\mathrm{O}$, et al. Interleukin-1 receptor antagonist (anakinra) treatment in patients with systemic-onset juvenile idiopathic arthritis or adult onset Still disease: preliminary experience in France. Ann Rheum Dis 2008;67:302-8.

18. Sandborg C, Mellins ED. A new era in the treatment of systemic juvenile idiopathic arthritis. N Engl J Med 2012;367:2439-40.

19. Ruperto N, Brunner HI, Quartier P, Constantin T, Wulffraat N, Horneff $\mathrm{G}$, et al. Two randomized trials of canakinumab in systemic juvenile idiopathic arthritis. N Engl J Med 2012;367:2396-406.

20. Pardeo M, Pires Marafon D, Insalaco A, Bracaglia C, Nicolai R, Messia V, et al. Anakinra in systemic juvenile idiopathic arthritis: a single-center experience. J Rheumatol 2015;42:1523-7.

21. Nigrovic PA, Mannion M, Prince FH, Zeft A, Rabinovich CE, Van Rossum MA, et al. Anakinra as first-line disease-modifying therapy in systemic juvenile idiopathic arthritis: report of forty-six patients from an international multicenter series. Arthritis Rheum 2011;63:545-55.

22. Vastert SJ, de Jager W, Noordman BJ, Holzinger D, Kuis W, Prakken BJ, et al. Effectiveness of first-line treatment with recombinant interleukin-1 receptor antagonist in steroid-naive patients with new-onset systemic juvenile idiopathic arthritis: results of a prospective cohort study. Arthritis Rheumatol 2014;66:1034-43

23. Ravelli A, Viola S, Ruperto N, Corsi B, Ballardini G, Martini A. Correlation between conventional disease activity measures in juvenile chronic arthritis. Ann Rheum Dis 1997;56:197-200.

24. Wallace CA, Ruperto N, Giannini E. Preliminary criteria for clinical remission for select categories of juvenile idiopathic arthritis. J Rheumatol 2004;31:2290-4.

25. Wallace CA, Giannini EH, Huang B, Itert L, Ruperto N. American College of Rheumatology provisional criteria for defining clinical inactive disease in select categories of juvenile idiopathic arthritis. Arthritis Care Res 2011;63:929-36.

26. Consolaro A, Bracciolini G, Ruperto N, Pistorio A, Magni-Manzoni $\mathrm{S}$, Malattia $\mathrm{C}$, et al. Remission, minimal disease activity and acceptable symptom state in juvenile idiopathic arthritis. Arthritis Rheum 2012;64:2366-74.

27. Consolaro A, Negro G, Chiara GM, Bracciolini G, Ferrari C, Schiappapietra B, et al. Defining criteria for disease activity states in non systemic juvenile idiopathic arthritis based on a three-variable juvenile arthritis disease activity score. Arthritis Care Res 2014;66:1703-9.

28. Magni-Manzoni S, Ruperto N, Pistorio A, Sala E, Solari N, Palmisani E, et al. Development and validation of a preliminary definition of minimal disease activity in patients with juvenile idiopathic arthritis. Arthritis Rheum 2008;59:1120-7.

29. Ravelli A, Consolaro A, Horneff G, Laxer RN, Lovell DJ, Wulffraat $\mathrm{N}$, et al. Treating juvenile idiopathic arthritis to target: recommendations of an international task force. Ann Rheum Dis 2018;77:819-28.

30. Minoia F, Consolaro A, Ravelli A. Filling the gap: toward a disease activity tool for systemic juvenile idiopathic arthritis. J Rheumatol 2018;45:3-5.

31. Consolaro A, Ravelli A. It is worth including assessment of disease activity state in juvenile arthritis clinical trials. Arthritis Care Res 2013;65:1207-10.

32. Lovell DJ, Giannini EH, Reiff AO, Kimura Y, Li S, Hashkes PJ, et al. Long-term safety and efficacy of rilonacept in patients with systemic juvenile idiopathic arthritis. Arthritis Rheum 2013;65:2486-96.

33. Shimizu M, Nakagishi Y, Yachie A. Distinct subsets of patients with systemic juvenile idiopathic arthritis based on their cytokine profiles. Cytokine 2013;61:345-8.

34. Ravelli A, Grom AA, Behrens EM, Cron RQ. Macrophage activation syndrome as part of systemic juvenile idiopathic arthritis: diagnosis, genetics, pathophysiology and treatment. Genes Immun 2012;13:289-98.

35. Minoia F, Davi S, Horne A, Demirkaya E, Bovis F, Li C, et al. Clinical features, treatment, and outcome of macrophage activation syndrome complicating systemic juvenile idiopathic arthritis: a multinational, multicenter study of 362 patients. Arthritis Rheumatol 2014;66:3160-9.

36. Nigrovic PA. Review: is there a window of opportunity for treatment of systemic juvenile idiopathic arthritis? Arthritis Rheumatol 2014;66:1405-13.

37. Lurati A, Teruzzi B, Salmaso A, Demarco G, Pontikaki I, Gattinara M. Macrophage activation syndrome during anti-IL-1 receptor therapy (anakinra) in a patient affected by systemic onset idiopathic juvenile arthritis. Pediatr Rheumatol Online J 2005;3:79-85.

38. Giannini EH, Ruperto N, Ravelli A, Lovell DJ, Felson DT, Martini A. Preliminary definition of improvement in juvenile arthritis. Arthritis Rheum 1997;40:1202-9. 